\title{
Determinação da carga externa no Pilates: comparação do footwork na chair e no reformer
}

\author{
External forces determination on Pilates: footwork on chair \\ and reformer comparison
}

\author{
Dr. Artur Bonezi ${ }^{1,2}$ \\ Dra. Renata Bona ${ }^{1}$ \\ Dra. Débora Cantergi ${ }^{2}$ \\ Dr. Jefferson Loss²
}

\section{RESUMO}

O método Pilates pode ser realizado com aparelhos dotados de molas. O conhecimento das forças envolvidas no exercício, bem como seu comportamento, ao longo da amplitude de execução permite uma melhor definição do exercício, contribuindo para uma escolha mais criteriosa e uma melhor prescrição do exercício. Assim, este estudo tem como objetivo determinar forças externas nos aparelhos chair e reformer e equiparar as forças externas do final da fase de extensão de quadril e joelho entre os aparelhos chair e reformer. Participaram 18 praticantes de Pilates há pelo menos seis meses. Foi empregado diagrama de corpo livre e equações de Newton-Euler para calcular a variação da força externa nos aparelhos chair e reformer no movimento footwork. Resultados são que há diferenças no comportamento das forças externas para o mesmo exercício, porém realizado nos dois diferentes equipamentos. Isso provavelmente implica diferenças na amplitude de movimento e nas estratégias de recrutamento, uma vez que a carga do equipamento final imposta era a mesma em ambos os aparelhos. Para concluir são apresentadas equação para determinar a carga externa final nos equipamentos chair e reformer. Essas informações podem ser levadas em consideração em um programa de condicionamento e reabilitação de Pilates.

\section{PALAVRAS-CHAVE}

Biomecânica; Pilates; Forças Externas; Estimativa.

\footnotetext{
${ }^{1}$ Laboratorio de Investigación en Biomecánica y Análisis del Movimiento, Centro Universitario de Paysandú, CENUR Litoral Norte, Universidad de la Republica Uruguay - Uruguay.

${ }^{2}$ Laboratório de Pesquisa do Exercício - Escola de Educação Física, Fisioterapia e Dança, Universidade Federal do Rio Grande do Sul - Brasil
} 


\section{ABSTRACT}

The Pilates method is performed by apparatus with springs. The knowledge of forces involved in an exercise, as well as their behavior throughout the range of execution allows a better exercise definition, contributing to a more careful choice and a better prescription of the exercise. So, the main goal of this study is to determine the external forces performed on chair and reformer apparatus and equate the external load end of the extension phase of hip and knee between the chair and the reformer. Eighteen women practice Pilates for at least six months. Free body diagram and Newton-Euler equations were used in order to calculate external force variation on chair and reformer apparatus during the footwork movement. Results indicate that there are differences on the behavior of external forces involved in the same exercise but performed on the two apparatus. This probably implies to differences in the range of motion and recruiting strategies, once the imposed final external load was the same for both apparatus. Concluding, equations are presented to determine the final external load on reformer and chair apparatus. This information might be considered in Pilates conditioning and rehabilitation program.

\section{KEYWORDS}

Biomechanics; Pilates; External Forces; Estimate. 


\section{INTRODUÇÃO}

A característica da resistência externa é um fator de grande influência na resposta dos músculos aos exercícios (KULIG et al., 1984) em virtude de torques externos crescentes, decrescentes, constantes ou mistos ao longo da amplitude de movimento (RASSIER et al., 1999).

A avaliação da resistência externa durante a excursão do gesto é importante para o entendimento do movimento, suas repercussões nas estruturas internas e aplicação na progressão de exercícios tanto na prática clínica como no contexto do treinamento.

A mensuração direta das forças externas requer instrumentos específicos, sendo necessárias adaptações nos aparelhos de Pilates. Esses instrumentos e adaptações nem sempre são de fácil aquisição e uso e adaptações necessitam de conhecimentos específicos. No Pilates algumas tentativas de mensurar as forças externas foram realizadas, por SELF et al. 1996 e BRODT et al. 2014 no aparelho reformer.

Uma alternativa para a mensuração direta é a análise indireta das forças, como a realizada no Pilates por SILVA et al. (2009) e LOSS et al. (2010) o qual avaliaram o torque de resistência externa do exercício de extensão de quadril no aparelho cadillac. Foi verificado que a resistência externa altera o padrão de recrutamento muscular. Em ambos os estudos supracitados a resistência externa foi dependente da altura de posicionamento da mola em relação à base do aparelho cadillac. Dessa maneira o conhecimento do comportamento das forças e torques, influenciam os exercícios e implicam em adaptações neuromusculares devido as diferentes exigências externas podendo alterar a característica e objetivo do exercício proposto (WINTER, 2005).

A ação das forças externas é uma característica impar dos equipamentos de Pilates, pois são utilizadas molas como resistência. Molas com diferentes cores indicam distintas constantes elásticas sendo que a constante elástica de uma mola depende da espessura da mola, do número e diâmetro das espiras e do material que a constitui (WILSON, 2004).

Existem estudos que mediram a força externa nos diferentes aparelhos utilizados no método Pilates (SELF et al., 1996; SILVA et al., 2009; LOSS et al.,
2010; BRODT et al., 2014). Contudo não há informações quanto à normalização e quantificação da resistência externa entre os aparelhos citados. Considerando que a resistência externa pode ser associada ao esforço empregado (SACCO et al., 2014), como por exemplo, no exercício de extensão de joelho e quadril, essa informação pode auxiliar aos profissionais da área na compreensão da sobrecarga que os exercícios impõem as articulações e músculos.

Dessa maneira, o presente estudo é o primeiro que se tem conhecimento com o intuito de normalizar a carga dos equipamentos e submeter todos os participantes ao mesmo esforço relativo entre aparelhos. Visando detalhar a estimativa das forças externas nos equipamentos de Pilates, o objetivo deste estudo foi (1) avaliar a variação da carga do equipamento nos aparelhos chair e reformer (2) equiparar a carga do equipamento chair, no final da fase extensão de quadril e joelho, à carga do reformer durante o movimento footwork do Pilates. Nós hipotetizamos que cada equipamento apresentará um comportamento mecânico distinto, devido as suas características impares, apesar do mesmo valor para carga final em ambos.

\section{MATERIAIS E MÉTODOS}

\section{Amostra}

Estudo foi composto por 18 mulheres praticantes do método Pilates (experiência $13 \pm 5$ meses) com idade de 28,9 $( \pm 6,4)$ anos, massa corporal de 55,4 $( \pm 4,6) \mathrm{kg}$, estatura de $1,64( \pm 0,05) \mathrm{m}$, e percentual de gordura de $18,5( \pm 3,6) \%$.

Este estudo de corte transversal seguiu as normas do conselho nacional de saúde (446/2012), todos os participantes foram informados dos procedimentos da pesquisa e assinaram o termo de consentimento livre e esclarecido o qual foi aprovado por meio do parecer 2008135 emitido pelo comitê de ética da universidade.

\section{Protocolo}

Primeiramente, foram coletadas a estatura e massa corporal dos sujeitos. Após aquecimento específico, realizado no próprio aparelho, os sujeitos foram posicionados sentados sobre a chair. 
A regulagem selecionada e o número de molas que foram utilizados na chair determinaram o cálculo da carga do equipamento para 10 repetições, detalhado nos próximos itens. Este equipamento precisava ser o primeiro a ser medido para determinar o cálculo da carga, pois nele o sujeito está em cadeia cinética aberta, o que não ocorre no reformer. Após foi calculada e igualdada a carga externa no equipamento reformer para as 10 repetições.

\section{Calibração das molas}

Previamente à coleta, foi determinada a constante elástica das molas dos aparelhos chair e reformer, procedimento conhecido como calibração, oriundo da engenharia mecânica e utilizado para análises biomecânicas (LOSS et al., 2002; WILSON, 2004). No presente estudo, a medida direta da constante elástica das molas foi obtida a partir de 12 medições. Após, foi efetuado um ajuste linear dos pontos experimentais o qual determinou a equação da reta e a respectiva constante elástica da mola. Para a mola do aparelho chair foi determinada uma constante elástica de $0,941 \mathrm{~kg} / \mathrm{cm}$ (com um $\mathrm{r}^{2}=0,9984$ ) e para as molas do aparelho reformer as constantes elásticas de 0,113 $\mathrm{kg} / \mathrm{cm}\left(\mathrm{r}^{2}=0,9868\right)$ e $0,232 \mathrm{~kg} / \mathrm{cm}\left(r^{2}=0,9813\right)$.

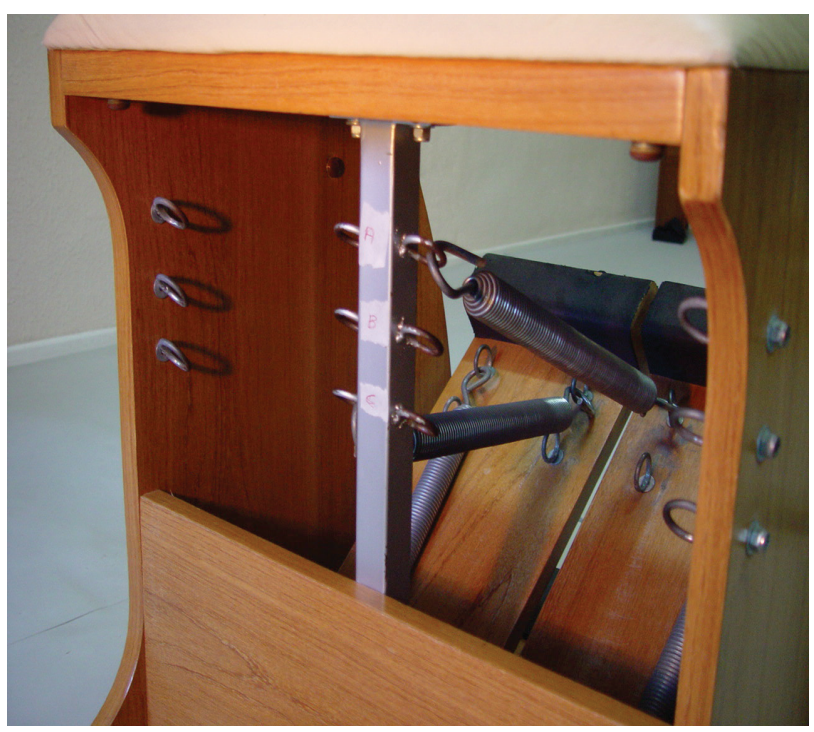

\section{Quantificação da força humana na chair}

Para calcular uma força devemos iniciar pelo diagrama de corpo livre. Este apresenta o segmento de interesse e as forças envolvidas. O aparelho chair possui diversas regulagens (figura $1 \mathrm{a}$ ) e quatro molas com a mesma constante elástica ( $K=0,941 \mathrm{~kg} / \mathrm{cm})$. A força que o indivíduo realiza no segmento mais distal (pé) foi chamada de força humana $\left(F_{\text {hum }}\right)$ na chair. Essa $F_{\text {hum }}$ foi determinada a partir do diagrama de corpo livre do segmento móvel do equipamento (figura 1b) conforme WINTER (2005), sendo que neste estudo se assumiu que a direção da $\mathrm{F}_{\text {hum }}$, no footwork na chair, é perpendicular ao apoio para os pés no equipamento (figura $1 \mathrm{~b}$ ). Na chair a $\mathrm{F}_{\text {hum }}$ e a carga do equipamento são o par ação e reação, ou seja, uma força de mesma magnitude e direção, mas sentido contrário (NEWTON, 2002).

Tanto no diagrama de corpo livre quanto nas equações são representadas as forças envolvidas. $O$ ângulo - determina a amplitude de movimento e o ângulo - a inclinação do vetor da força elástica. Considera-se que os efeitos inerciais são negligenciáveis em movimentos lentos, velocidades angulares meno-

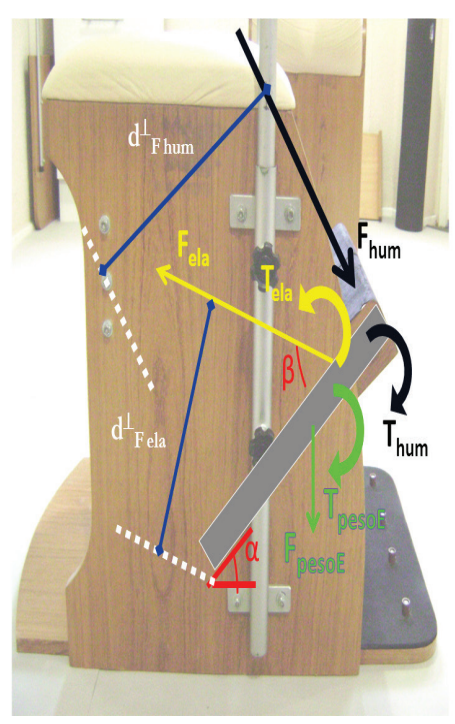

Figura 1: Aparelho chair: (a) visão posterior das regulagens e (b) diagrama de corpo livre do segmento móvel da chair. Estão representadas a força humana $\left(F_{\text {hum }}\right)$, a força elástica $\left(F_{\text {ela }}\right)$ e suas respectivas distâncias perpendiculares $\left(d^{\perp}\right.$ Fhum $)$ e $\left(d \perp_{\text {Fela }}\right)$. Também é apresentado o torque elástico $\left(T_{\text {ela }}\right)$ o torque peso do apoio para os pés equipamento $\left(T_{\text {pesoE }}\right)$ e a força peso do equipamento $\left(\mathrm{F}_{\text {peso: }}\right)$. Foi assumido que a $\mathrm{F}_{\text {hum }}$ é perpendicular a base do equipamento. $\mathrm{O}$ ângulo $\alpha$ determina amplitude de movimento e $\beta$ representa a inclinação do vetor da força elástica 
res que 30\%, (LOSS; CANDOTTI, 2008). Partindo da premissa que o sistema está em equilíbrio (equações de movimento Newton-Euler) é possível escrever a equação 1 :

$$
\tau_{\text {hum }}=\tau_{\text {ela }}-\tau_{\text {peso }}
$$

\section{Equação 1}

Reescrevendo a equação 1 , obteve-se a equação 2 :

$$
F_{\text {hum }} \text { Chair }=\frac{F_{\text {ela }} \cdot d_{F_{\text {ela }}^{\perp}}^{\perp}}{d_{F_{\text {hum }}}^{\perp}}-\tau_{\text {peso E }}
$$

\section{Equação 2}

onde,

$\tau_{h u m} \tau_{h u m} \Gamma_{e x}$ é o torque (momento) humano;

$\tau_{\text {ela }} \tau_{\text {ela }} \Gamma_{\text {ela é o torque (momento) elástico; }}$

$\tau_{\text {pesoE }} \tau_{\text {pesoE }}$ é o torque (momento) do peso do apoio para os pés do equipamento;

$F_{\text {hum }}$ Chair $F_{\text {hum }}$ Chair é a magnitude da força humana gerada na chair;

$F_{\text {ela }} F_{\text {ela }}$ é a força elástica proporcionada pelas molas;

$d_{F_{\text {ela }}}^{\perp} d_{F_{\text {ela }}}^{\perp}$ é a distância perpendicular força elástica;

$d_{F_{h u m}}^{\perp} d_{F_{h u m}}^{\perp}$ é a distância perpendicular da força humana;

Após o individuo realizar a sua máxima força na chair, para realizar 10 repetições respeitando os princípios do método Pilates, foi verificada a posição das molas no aparelho. Além de variar o tipo de mola também era possível variar o local de fixação da mola (figura 1a). o que poderia determinar uma pré-distensão na mola no início do movimento. Com essas informações, ocorreu a determinação das forças envolvidas.

Com auxílio de um goniômetro manual e fita métrica, foi possível medir diretamente os ângulos de inclinação das molas, as distâncias e após calcular as distâncias perpendiculares da força elástica e da $\mathrm{F}_{\text {hum }}$. Essas mensurações foram realizadas para todas as seis combinações possíveis de posicionamento de cada mola. A partir de relações trigonométricas, das forças e momentos relacionados e o conhecimento da variação das distâncias perpendiculares envolvidas ao longo da amplitude de movimento foi possível a determinação da $\mathrm{F}_{\text {hum }}$ ao longo de toda amplitude do aparelho chair (SILVA et al., 2009; MELO et al., 2011;). O valor da $F_{\text {hum }}$ final - na máxima amplitude do exercício - foi utilizado para equiparar a $F_{\text {hum }}$ final do reformer através de rotinas em Matlab.

\section{Quantificação da força humana no reformer}

$A F_{\text {hum }}$ no reformer representa o para ação e reação a carga do equipamento (NEWTON, 2002). O reformer possuí cinco molas (figura 2a) e no aparelho analisado três apresentavam mesma constante elástica $(\mathrm{K}=0,113 \mathrm{~kg} / \mathrm{cm})$ e duas molas apresentavam uma constante maior $(\mathrm{K}=0,232 \mathrm{~kg} / \mathrm{cm})$.

Essas molas podem ser configuradas com diferentes comprimentos iniciais. No nível denominado de I a mola está em seu comprimento original, sem qualquer alteração no seu tamanho; no nível II a mola está com $5 \mathrm{~cm}$ de pré-estiramento; no nível III a mola está submetida a $10 \mathrm{~cm}$ de pré-estiramento. Neste estudo todas as possibilidades de combinações entre as 5 molas (considerados os 2 tipos de constantes elásticas) e as 3 regulagens foram testadas e então a melhor configuração foi utilizadas para cada indivíduo (figura 2 a). 

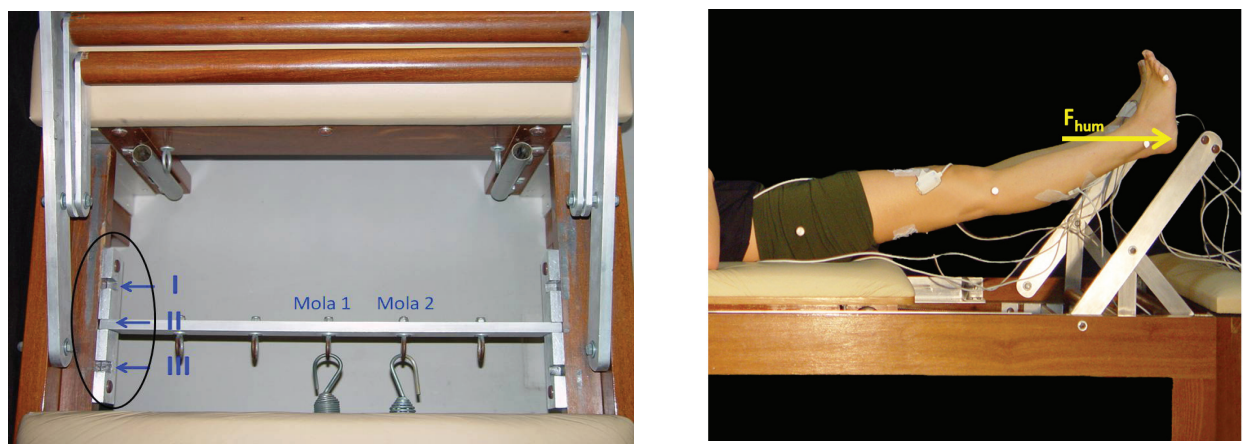

Figura 2: Aparelho reformer: (a) regulado com duas molas, de diferentes constantes elásticas, no nível II e (b) sentido da força humana $\left(F_{\text {hum }}\right)$ no segmento pé.

A literatura já havia reportado que a massa e a estatura dos indivíduos têm influência na carga do equipamento do reformer (SELF et al., 1996). Conhecendo a estatura do indivíduo - a qual determinará a variação de comprimento máximo da mola - e as combinações de molas do aparelho a $F_{\text {hum }}$ no reformer, foi calculada utilizando a equação 3 :

onde,

$$
F_{\text {hum }} \operatorname{Refor}=(n) K_{1} \cdot x+(m) K_{2} \cdot x
$$

Equação 3

$F_{\text {hum }}$ Refor $F_{\text {hum }}$ Refor é a magnitude da força humana gerada no reformer;

$n n$ é o número de molas ( 1 a 2 ) de constante elástica $K_{1} K_{1}$;

$K_{1} K_{1}$ é a constante elástica de $\mathrm{k}=0,232 \mathrm{~kg} / \mathrm{cm}$;

$m m$ é o número de molas (1 a 3) de constante elástica $K_{2} K_{2}$;

$K_{2} K_{2}$ é a constante elástica de $\mathrm{k}=0,113 \mathrm{~kg} / \mathrm{cm}$;

$x x$ é a variação de comprimento da mola.

$\mathrm{F}_{\text {hum }}$ Reformer foi determinada por rotina desenvolvida em Matlab. A rotina busca entre as diversas possibilidades de molas, número de molas, níveis da mola e estatura de cada individuo, a combinação que mais se aproxima do valor da $\mathrm{F}_{\text {hum }}$ produzido na Chair para cada indivíduo analisado. Dessa maneira a quantificação da $\mathrm{F}_{\text {hum }}$ à qual o indivíduo foi submetido no segmento distal pé no final do exercício (figura 2b) deverá ser a mesma nos dois aparelhos (ZATSIORSKY, 2002).

\section{Tratamento estatístico}

A análise estatística do presente estudo foi realizada no software SPSS 17.0, o nível de significância adotado, em todos os testes, foi de $p<0,05$. A normalidade foi testada através do Teste de Shapiro-

-Wilk para todas as variáveis. Após a hipótese de normalidade ser aceita, a homogeneidade dos dados foi verificada com o Teste de Levene. 0 teste $t$ Student para amostra emparelhadas foi utilizado para comparar os valores da $F_{\text {hum }}$ entre os dois aparelhos.

\section{RESULTADOS}

Na figura 3 é apresentado o comportamento da $\mathrm{F}_{\text {hum }}$ nos aparelhos chair e reformer durante o movimento footwork em relação ao ângulo de flexão da articulação do joelho (a) e quadril (b), conforme convencionado (KULIG et al., 1984). Ambas as curvas apresentaram característica linear crescente, devido à característica elástica das molas, contudo o comportamento foi diferente, devido às diferentes amplitudes de movimento dos exercícios. 

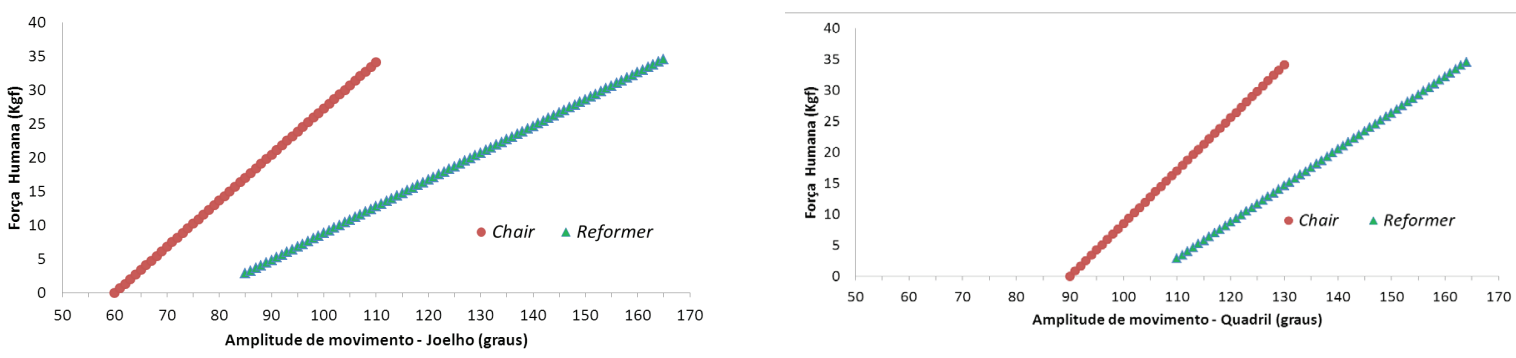

Figura 3: Comportamento da força humana nos aparelhos chair e reformer durante o movimento footwork. a) em relação articulação do joelho. b) em relação articulação do quadril

(dados de um indivíduo representativo da amostra).

A tabela 1 apresenta os valores de $\mathrm{F}_{\text {hum }}$ da chair e reformer calculados para cada indivíduo além dos valores médios da $\mathrm{F}_{\text {hum }}$ da chair e da $\mathrm{F}_{\text {hum }}$ do reformer no final da amplitude, bem como a diferença entre os aparelhos. Tal diferença representa a variação na determinação da $F_{\text {hum }}$ entre os aparelhos. Não foram encontradas diferenças significativas entre os valores da $F_{\text {hum }}$ nos dois aparelhos $(p>0,05)$. A moda das combinações utilizada na chair foi duas molas na regulagem A e C (mais alta e mais baixa) conforme figura $1 \mathrm{a}$. No reformer a moda da quantidade e da posição das molas utilizadas foi: quatro molas (duas com constantes elásticas de 0,113 $\mathrm{kg} / \mathrm{cm}$ e duas de $0,232 \mathrm{~kg} / \mathrm{cm}$ ) na regulagem $B$ (intermediaria) figura $2 \mathrm{a}$.

Tabela 1 - Valores individuais, médios e desvios padrão determinados para a força humana $\left(\mathrm{F}_{\text {hum }}\right)$ na chair e calculados para o reformer no final da amplitude movimento. Também é apresentado o valor (individual, médio e desvio padrão) da diferença entre os valores da $\mathrm{F}_{\text {hum }}$ Chair e Reformer (Dif C-R) calculado para cada indivíduo $(p>0,05)$.

\begin{tabular}{ccccc}
\hline & $\mathrm{F}_{\text {hum }}$ Chair (kgf) & $\mathrm{F}_{\text {hum }}$ Reformer (kgf) & Dif C-R (kgf) \\
\hline Indivíduo 1 & 31,26 & 31,49 & 0,23 \\
Indivíduo 2 & 31,27 & 31,49 & 0,22 \\
Indivíduo 3 & 26,55 & 27,38 & 0,83 \\
Indivíduo 4 & 31,26 & 31,22 & $-0,04$ \\
Indivíduo 5 & 39,73 & 40,75 & 1,02 \\
Indivíduo 6 & 34,08 & 34,23 & 0,15 \\
Indivíduo 7 & 23,73 & 23,96 & 0,23 \\
Indivíduo 8 & 26,55 & 26,01 & $-0,54$ \\
Indivíduo 9 & 23,73 & 23,44 & $-0,29$ \\
Indivíduo 10 & 28,44 & 28,38 & $-0,06$ \\
Indivíduo 11 & 26,55 & 26,91 & 0,36 \\
Indivíduo 12 & 26,55 & 26,96 & 0,41 \\
Indivíduo 13 & 31,26 & 31,96 & 0,70 \\
Indivíduo 14 & 34,08 & 34,23 & 0,15 \\
Indivíduo 15 & 36,91 & 36,49 & $-0,42$ \\
Indivíduo 16 & 28,44 & 28,43 & $-0,01$ \\
Indivíduo 17 & 23,73 & 23,96 & 0,23 \\
Indivíduo 18 & 28,44 & 28,43 & $-0,01$ \\
\hline Média & $\mathbf{2 9 , 6 5 ( \pm 4 , 6 8 )}$ & $\mathbf{2 9 , 8 4}( \pm 4,78)$ & $\mathbf{0 , 1 9}( \pm 0,41)$ \\
\hline
\end{tabular}




\section{DISCUSSÃO}

O objetivo do presente estudo foi calcular a variação da $\mathrm{F}_{\text {hum }}$ nos aparelhos chair e reformer durante o movimento footwork do Pilates e equiparar a $F_{\text {hum }}$ final na fase extensão de quadril e joelho em ambos os aparelhos.

Autores ressaltam a importância que diferentes amplitudes de movimento podem ter sobre variações do mesmo exercício, como agachamento e leg press (ESCAMILLA et al., 1998; ESCAMILLA et al., 2001; FRY et al., 2003) em relação à ativação muscular, sobrecarga (resistência externa) e lesões das articulações envolvidas.

A relação força-comprimento é afetada pela variação de amplitude de movimento e a produção de força muscular está relacionada a uma demanda funcional, ou seja, um processo de adaptabilidade (RASSIER et al., 1999; BABAULT et al., 2003). No exercício footwork a $\mathrm{F}_{\text {hum }}$ é produzida pelos mesmos grupos musculares tanto na chair como no reformer. Entretanto as excursões dos principais músculos durante o movimento são provavelmente diferentes, sendo a amplitude de movimento maior no reformer do que na chair. Sabe-se que diferentes comprimentos musculares influenciam na organização e função muscular, de músculos sinergistas, controlada pelo sistema nervoso central (DISSELHORST et al., 2009). Esse conhecimento pode influenciar a escolha do equipamento ao visar treinamento, prevenção ou reabilitação física.

Fora do ambiente do Pilates FRY et al. (2003) utilizando a abordagem indireta para quantificação das forças compararam os torques resultantes no joelho e quadril em duas variações da técnica de agachamento. Utilizando cinemetria, parâmetros antropométricos e diagrama de corpo livre para determinação dos torques os autores concluíram que o torque no joelho é maior que no quadril na técnica de agachamento livre enquanto na técnica de agachamento restrito o resultado foi inverso. Também houve diferenças nas amplitudes do exercício, entre as técnicas. A partir desses dados se concluiu que alterações na técnica promoveram diferentes padrões de sobrecarga articular, como forças de compressão e cisalhamento, que são fatores de risco para lesões (FRY et al., 2003).
FOLLAND e MORRIS (2006) examinaram que máquinas diferentes, para exercitar o mesmo grupamento muscular de extensores de joelho, apresentam curvas com características mecânicas distintas (comportamento de torque externo) e influenciam nas exigências musculares específicas. Isso corrobora com os dados do presente estudo uma vez que o equipamento é um fator gerador de influência na demanda muscular (KULIG et al., 1984; FRY et al., 2003; FOLLAND; MORRIS, 2006).

Da literatura consultada, poucos estudos quantificaram a resistência externa no Pilates. Self et al. (1996) verificaram, entre outros parâmetros, a influência de duas ou quatro molas na força mensurada fornecendo informações cinéticas precisas. A tabela 1 apresenta os valores médios estimados indiretamente para a $\mathrm{F}_{\text {hum }}$ no reformer do presente estudo $(29,84 \pm 4,78$ kgf), os quais estão próximos aos mensurados na literatura através de célula de carga acopladas ao reformer $(30,41 \pm 1,68 \mathrm{kgf})$ de cinco muIheres utilizando quatro molas durante movimento similar (SELF et al. 1996). Já Silva et al. (2009) e MELO et al. (2011) determinaram o torque de resistência ao longo do exercício de extensão de quadril realizado no cadillac concluindo que o posicionamento das molas influencia na característica do torque.

A presente proposta foi capaz de quantificar as forças das molas, calcular a $\mathrm{F}_{\text {hum }}$ e equiparar as cargas estando embasada em fatos bem descritos na literatura (LOSS et al., 2002; FRY et al., 2003; LOSS; CANDOTTI, 2008, MELO et al., 2011, SACCO et al., 2014) e, apesar de suas limitações (estimativa da força elástica e cálculos indiretos das forças), se mostrou uma ferramenta eficaz e interessante de ser aplicada no Pilates. Dessa maneira, o estudo busca elucidar como ser obtida a força das molas e com as equações apresentadas facilmente pode-se calcular a força humana. Essa estimativa é importante e exequível em equipamentos nos estúdios e clínicas de Pilates, pois permitiria maior controle do esforço que esta sendo exigido dos praticantes.

\section{CONCLUSÃO}

Foi possível avaliar a variação da carga dos equipamentos chair e reformer. Após se equiparar a car- 
ga final entre eles durante um exercício de flexão de joelho e quadril do Pilates (footwork) verificou-se que há diferenças no comportamento da resistência externa oferecido pelos equipamentos devido as suas características mecânicas. Esses achados podem contribuir para a prescrição de treinamento físico, prevenção de lesões e reabilitação física, através da escolha do equipamento, conhecimento da amplitude de movimento e carga selecionada.

\section{REFERÊNCIAS}

BABAULT, N.; POUSSON, M.; MICHAUT, A.; VAN HOECKE, J. Effect of quadriceps femoris muscle length on neural activation during isometric and concentric contractions. Journal of Applied Physiology, v. 94, n. 3, p. 983-990, 2003.

BRODT, G.A.; CANTERGI, D.; GERTZ, L. C. ; LOSS, J. F. An instrumented footbar for evaluating external forces in Pilates. Journal of Applied Biomechanics, v. 30, n. 3, p. 483490, 2014

DISSELHORST-KLUG, C.; SCHMITZ-RODE, T.; RAU, G. Surface electromyography and muscle force: limits in sEMG-force relationship and new approaches for applications. Clinical biomechanics, v. 24, n. 3, p. 225-235, 2009.

ESCAMILLA, R. F.; FLEISIG, G. S.; ZHENG, N.; LANDER, J.; BARRENTINE, S. W.; ANDREWS, J.; BERGEMANN, B. W.; MOORMAN, C. Effects of technique variations on knee biomechanics during the squat and leg press. Medicine and Science in Sports and Exercise, v. 33, n. 9, p.1552 -1566, 2001

ESCAMILLA, R. F.; FLEISING, G.; BARRENTINE, S. W.; WILK, K. E.; ANDREWS, J. R. Biomechanics of the knee during closed kinetic chain and open kinetic chain exercise. Medicine and Science Sport and Exercise, v. 30, n. 4, p. 556569,1998

FOLLAND, J.; MORRIS, B. Variable-cam resistance training machines: do they match the angles-torque relationship in humans? Journal of Sports Sciences, v. 1, n. 1, p. 1-7, 2007.

FRY, A.; SMITH, C.; SCHILLING, B. Effect of knee position on hip and knee torques during the barbell squat. Journal of Strength and Conditioning Research, v. 17, n. 4, p. 629 $633,2003$.

KULIG, K.; ANDREWS, J.; HAY, J. Human strength curves. Exercise and Sports Sciences Reviews. v. 12, p. 417-466, 1984.

LOSS, J. F.; CANDOTTI, C. T. Comparative study between two elbow flexion exercises using the estimated resultant mus- cle force. Revista Brasileira de Fisioterapia, v. 12, n. 6, p. 502-510, 2008.

LOSS, J.F.; MELO, M.D.O.; ROSA, C.H.; SANTOS, A.B.D.; TORRE, M.L.; SILVA, Y.O.D. Electrical Activity of external obliques and multifidus muscles during the hip flexion-extension exercise performed in cadillac with differents adjustment for springs and individual positions. Revista Brasileira de Fisioterapia, v.14, n.6, p.510-517, 2010.

LOSS, J. F.; KOETZ, A.; SOARES, D.; SCARRONE, F.; HENNEMANN, V.; SACHARUK, V. Quantificação da resistência oferecida por bandas elásticas, Revista Brasileira de Ciências do Esporte, v. 24, n. 1, p. 61-72, 2002.

NEWTON, Isaac. Principia - Princípios Matemáticos De Filosofia Natural - Livro 1. $2^{\mathrm{a}}$ ed, São Paulo: Edusp, 2002. 325p. Originalmente publicado como Philosophiae Naturalis Principia Mathematica em 1687. Tradução da versão inglesa realizada por Andrew Motte em 1729 diretamente do original.

MELO, M. O.; GOMES, L. E.; SILVA, Y. O.; SANTOS, A. B.; LOSS, J. F. Resultant muscular force and resistance torque assessment during hip extension exercise in pilates and its implications on prescription and progression. Revista Brasileira de Fisioterapia, v.15, n.1, p.23-30, 2011.

RASSIER, D. E; MACINTOSH, B. R.; HERZOG, W. Length dependence of active force production in skelectal muscle. Journal of Applied Physiology, v. 86, n. 5, p.1445-1457, 1999.

SACCO, I. ; MORI, E.; MARCONI, N.; QUEIROZ, B.; PEREIRA, I. Electromyographic assessment of trunk and shoulder muscles during a Pilates pull-up exercise. Motriz : Revista de Educação Física v. 20, p. 206-212, 2014.

SELF, B. P.; BAGLEY, A. M.; TRIPLETT, T. L.; PAULOS, L. E. Functional biomechanical analysis of the pilates-based reformer during demi-plie movements Journal of Applied Biomechanics, v. 12, n. 3, p. 326-337, 1996.

SILVA, Y. O.; MELO, M. O.; GOMES, L. E.; BONEZI, A.; LOSS, J. F. Analysis of the external resistance and electromyographic activity of hip extension performed according to the Pilates method. Revista Brasileira de Fisioterapia, v. 13, n. 1, p. 82-88, 2009.

WILSON, J. D. Physics Laboratory Experiments. $6^{\mathrm{a}}$ ed. Houghton: Mifflin Company, 2004. 481p.

WINTER, D. Biomechanics and Motor Control of Human Movement. $3^{\mathrm{a}}$ ed. New Jersey: John Wiley \& Sons, 2005. $277 p$.

ZATSIORSKY, V. M. Kinetics of Human Motion. $1^{\text {a }}$ ed. Champaign: Human Kinetics, 2002. 672p. 